Fibronectin/thermo-responsive polymer scaffold as a dynamic ex vivo niche

\title{
for mesenchymal stem cells
}

Laura Ramalho $^{1,3}$, Salima Nedjari ${ }^{1}$, Roberto Guarino ${ }^{4}$, Firas Awaja ${ }^{2,5,6}$, Dencho Gugutkov ${ }^{1}$, George Altankov ${ }^{1,2, * *}$

${ }^{1}$ ICREA and ${ }^{2}$ Institute for Bioengineering of Catalonia (IBEC) Barcelona, Spain

${ }^{3}$ Institute of Biophysics and Biomedical Engineering, Faculty of Sciences, University of Lisbon, Portugal

${ }^{4}$ École Polytechnique Fédérale de Lausanne (EPFL), Swiss Plasma Center (SPC), CH-5232

Villigen PSI, Switzerland

${ }^{5}$ Present address: Department of Orthopaedic Surgery, Medical University Innsbruck, Austria and Regenerative Medicine Institute (REMEDI) and Centre for Research in Medical Devices, (CÚRAM) at National University of Ireland, Galway, Ireland

${ }^{6}$ Engmat Ltd., Clybaun Road, Galway, Ireland

** Corresponding author: altankov@abv.bg

** Present address: Associate Member Institute for Biophysics and Biomedical Engineering, Bulgarian Academy of Sciences, Sofia, Bulgaria 


\section{ABSTRACT}

In this paper, we created a dynamic adhesive environment (DAE) for adipose tissue-derived mesenchymal stem cells (ADMSCs) cultured on smart thermo-responsive substrates, i.e., poly (N-isopropyl acrylamide) (PNIPAM), via introducing periodic changes in the culture temperature. We further explored the particular role of adsorbed fibronectin (FN), an important cell adhesive protein that was recently attributed to the recruitment of stem cells in the niche. The engineered FN/PNIPAM DAE system significantly increased the symmetric renewal of ADMSCs, particularly between passages 7 and 9 (p7-p9), before it dropped down to the level of the control (FN-coated TC polystyrene). This decline in the growth curve was consistent with the increased number of senescent cells, the augmented average cell size and the suppressed FN matrix secretion at late passages (p10-p12), all of them characteristic for stem cells ageing, which equivocally tended to slow down at our DAE system. FN supported also the osteogenic response of ADSC (apart from the previous observations with plain PNIPAM substrata) indicated by the significant increase of Alkaline Phosphatase (ALP) activity at day $7^{\text {th }}$ and $14^{\text {th }}$. The minimal changes in the Ca deposition, however, suggest a restricted effect of DAE on the early osteogenic response of ADMSCs only. Thus, the engineering of niche-like DAE involving FN, uncovers a new tissue engineering strategy for gaining higher amount of functionally of active stem cells for clinical application.

\section{KEYWORDS}

Extracellular matrix; mesenchymal stem cells; stem cell niche; ageing; self-renewal; osteogenic differentiation; dynamic adhesive environment; thermo-responsive polymer; PNIPAM. 


\section{INTRODUCTION}

Adult stem cells (ASCs) are major contributors for the growth and regeneration of tissues after damage or ageing [1-5]. They have great therapeutic potential due to the unique ability to differentiate into multiple cell lineages thus assuring diverse tissues repair $[3,6]$. However, most cell-based therapies need high amount of ASCs, which is an obstacle as stem cells are generally rare population [2,7]. It requires long propagation in vitro which leads to the loss of their differentiation potency presumably due to ageing, or other not well characterized factors $[5,8]$. Therefore, the obtaining control over the ASCs behaviour both in vitro and in vivo is a critical step toward the development of regenerative therapies and for the treatment of ageing [8-11].

ASCs fate is tightly regulated by complex spatiotemporal signals originating from the surrounding physical and chemical milieu - the stem cell niche [2,12]. Consequently, precise engineering of the niche environment is a way for controlling their behaviour [12,13]. Factors that are currently recognized as determiners of ASCs fate are the soluble growth factors and cytokines, as well as the physical interaction with the surrounding extracellular matrix (ECM) [14] and also other cells $[1,15]$. Two major parameters that reflect the unique functionality of stem cells are related to their capability for symmetric self-renewal [16] and to their competence to differentiate into multiple lineages [17]. Symmetric self-renewal is a mechanism by which each stem cell creates two identical daughter cells leading to the expansion of undifferentiated cell pool required for repair [1]. In vivo, the self-renewal is controlled by the niche microenvironment. Instead, in vitro (for example, when one need to generate larger cell mass) it is often swapped by a non-regulated symmetric division [17], characterized by a limited number of passages and decline in the cell functionality due to an accelerated ageing $[3,10,11]$. 
One strategy for engineering particular niches relies on using biomaterials constrained to mimic the natural cell-ECM interaction $[13,18]$. Selective binding of matrix components or to specially engineered cell adhesive sequences of collagen and laminin has been recently explored [19-21], presuming that stem cells will recognize the adhesive environment and will respond to it specifically $[21,22]$. The particular role of fibronectin (FN) is sparsely investigated, though this glycoprotein is probably the best studied adhesive factor triggering cell adhesion to foreign substrates [23]. FN exists both in soluble (dimeric) form and as insoluble polymer in the ECM. Its important role for the recruitment of stem cell in the niche [14] and for their maintenance in an undifferentiated state was only recently recognized [23]. The dynamic aspects of these adhesive interactions however have not been explored yet.

An obstacle for engineering of natural niche settings is that the ECM itself represents a dynamic adhesive environment (DAE) due to its continuous remodelling by the cells $[24,25]$. Considering this, we recently hypothesised that stem cells in the niche are exposed to a frequently altered mechanical stimuli that will strongly affect their behaviour, involving the most important decisions they have to take, e.g., to stay in the niche for self-renewal, or to move away and start differentiation at a distant place. Consequently, we postulate that engineering such a DAE ex vivo will provide insights for the better understanding of ASCs behaviour, and consequently for guiding their fate [26].

Another important issue relates to the source of ASCs that are suitable for such investigations. Recently, adipose tissue-derived mesenchymal stem cells (ADMSCs) gain distinct interest due to their exceptionally high amount in oily depots (about 4\%) and in the same time providing minimally invasive conditions for harvesting $[27,28]$. On the other hand, many recent studies identify ADMSCs as important precursors of multiple organ regeneration $[2,3]$ due to the differentiation into tri-germ lineages including, e.g., osteocytes, adipocytes, 
neural cells, vascular endothelial cells, cardiomyocytes, pancreatic $\beta$-cells, and hepatocytes [3]. This make them very useful for clinical applications.

A promising kind of materials that could change our understanding for the niche microenvironment is the stimuli-responsive polymers [29]. These materials undergo radical changes of their internal structure upon external stimuli (e.g., temperature, $\mathrm{pH}$, light) and may be used as "ON/OFF"-switches between different functions [30], but in the same time providing potential for the introduction of reverse (dynamic) effects [26]. The thermoresponsive polymer poly (N-isopropyl acrylamide) (PNIPAM) has been widely used to induce cell attachment/detachment by modifying the surface wettability with only subtle changes of the culture temperature [31-34]. In general, cells are unable to recognise foreign materials [13] but they do recognize the adsorbed proteins from the surrounding medium [30]. Hence the "classical" model of cell-substratum interaction requires adsorption of an adhesive protein, for example, fibronectin $(\mathrm{FN})$ or vitronectin [31], though any other purified ECM protein might trigger cell attachment [22,32] once is recognised by cell surface integrins. Decreasing the temperature of PNIPAM from $37^{\circ} \mathrm{C}$ to room temperature, i.e., below the LCST (Lower Critical Solution Temperature) increases the swelling of the material and cells are released from the surface together with the involved adhesive protein without the need for digestive enzymes $[32,34]$. However, the reversibility of this phenomena that might provide for the creation of a DAE for stem cells is not sufficiently explored, though clearly demonstrated in our recent investigation [26].

In fact, this paper represents a follow-up study based on our previous observation that periodic DAE cycles, causing reversible attachment/detachment of ADMSCs, dramatically alter their differentiation potential when cultured on plane PNIPAM in "classical" serum containing medium [26]. Here, we explore the particular role of adding FN to the system and its impact on the osteogenic differentiation. $\mathrm{FN}$ is an important ECM protein that play crucial 
role in cell-biomaterial interaction. It has been recently shown to be directly involved in the maintaining of stem cells fate in the niches [14,23]. FN is not present in the standard serum preparations as it binds to fibrin during the clotting of plasma [35,36]. Consequently, $\mathrm{FN}$ is absent as external adhesive factor in the regular cell culture conditions. Here we evaluate its specific effect under DAE by means of single-protein adsorption system.

\section{MATERIALS AND METHODS}

\section{Cells}

Human ADMSCs (passage 1) were obtained from Lonza Biowhittaker (Verviers, Belgium). After thawing they were allowed to grow for one passage before starting the studies using standard $25 \mathrm{~cm}^{2}$ flask (Thermo Scientific, 136196). For all experiments the cells were maintained in DMEM/F12 (1:1) supplemented with 1\% GlutaMAX ${ }^{\mathrm{TM}}$ (Gibco, 31331-028), $1 \%$ Antibiotic-Antimycotic solution (Gibco, 15240-062) and 10\% Fetal Bovine Serum (FBS) (Gibco, 10270-106), and incubated at $37{ }^{\circ} \mathrm{C}, 5 \% \mathrm{CO}_{2}$ and $98 \%$ humidity. The medium was replaced every $2^{\text {nd }}$ day.

\section{Cell sub-culturing}

At the end of each passage (5 days) the cells were harvested using Trypsin/EDTA ( 5 min) and seeded at a density of $2 \times 10^{4}$ cells $/ \mathrm{cm}^{2}$ on commercially available PNIPAM-grafted substrata or regular TC polystyrene, as specified below. $60 \mathrm{~mm}$ PNIPAM-grated Petri dishes, used for the morphological studies, and 24 well PNIPAM culture plates (for all other subculture experiments) were provided by Thermo-Fisher Nunc UpCell ${ }^{\mathrm{TM}}$ (Cat. No. 174903 and 17489, respectively). As controls, the same sized dishes from regular TC polystyrene (SigmaAldrich, P5481 500EA) were used. All substrata (both control and PNIPAM) were pre-coated 
with $20 \mu \mathrm{g} / \mathrm{ml}$ of human plasma fibronectin (FN) (Sigma-Aldrich, F2006) for $30 \mathrm{~min}$ at $37{ }^{\circ} \mathrm{C}$, then washed with PBS before adding the cells.

\section{Thermo-cycling}

After 24 h of cell seeding, both PNIPAM and control polystyrene samples were exposed to thermo-cycling using a custom made stainless steel thermo-plate with build in channels for circulating water. It was fitted to ultra-thermostat KH-4 (Biometra, 043-300) providing for both heating $\left(37^{\circ} \mathrm{C}\right)$ and cooling $\left(20^{\circ} \mathrm{C}\right)$ regimes. A preliminary study [26] indicated as optimal the temperature cycle of $30 \mathrm{~min}$ cooling (from $37^{\circ} \mathrm{C}$ to $20^{\circ} \mathrm{C}$ ) and $30 \mathrm{~min}$ heating (from $20^{\circ} \mathrm{C}$ to $37^{\circ} \mathrm{C}$ ) accepted as standard procedure that we applied twice a day (morning and evening).

\section{Cells passageing}

Five days after seeding, the cells were detached with Tripsin/EDTA, followed by a gentle pipetting aimed to obtain single cells. The cells were counted (see below) and then seeded again in new wells at the same initial density (i.e., $2 \times 10^{4}$ cells $/ \mathrm{cm}^{2}$ ) for the subsequent passage. The rest of ADMSCs were used for determining the overall morphology, FN matrix secretion and senescence (see below).

\section{Average cell size}

Life Technologies Countess 11 Automated Cell Counter AMQAX1000 (purchased by ThermoFisher Scientific) was used to count the cells at each passage and to measure the average cells size in suspension obtained from three independent samples.

\section{Proliferation and cell doubling assay}

Cell proliferation was assayed by direct counting of cells number at 5th day of passageing using automated cell counter. To assure accuracy of the measurement, counting 
was performed in triplicates and frequently verified by counting with Neubauer chamber under phase contrast. The calculation of the Cumulative Population Doubling Time (CPDT) was performed according to [37] as follows:

$$
\begin{gathered}
\mathrm{CPDT}=\frac{\mathrm{CT}}{\mathrm{CPDN}} \\
\mathrm{CPDN}={ }^{\ln \left(N_{f} / N_{i}\right) / \ln 2}
\end{gathered}
$$

where CPDN is the Cumulative Population Doubling Number, $N_{i}$ and $N_{f}$ are the initial and final cell numbers, respectively, and CT is the culture time.

\section{Overall cell morphology and visualization of focal adhesion complexes}

To follow the effect of temperature on cell morphology, ADMSCs were seeded as above on FN coated PNIPAM and control Petri dishes, for $24 \mathrm{~h}$. The samples were then fixed with 4\% paraformaldehyde (Pareac, 141451-1210) and processed for immunofluorescence. To follow the focal adhesion formation and actin cytoskeleton reorganization, fixed samples were permeabilized with $0.5 \%$ Triton-X 100 (Sigma-Aldrich) and saturated with 1\% BSA for 20 min to avoid unspecific binding before stained with a monoclonal anti-vinculin antibody (Sigma-Aldrich, V9264) followed by goat anti-mouse IgG AlexaFluor 555-conjugated secondary antibody (Life Technologies, A-21422) both incubated for $30 \mathrm{~min}$ at $37{ }^{\circ} \mathrm{C}$. The mixture of secondary antibody contained FITC-Phalloidin 488 (Life Technologies, A12379) and Hoechst (Invitrogen, 34580) for simultaneous counterstaining of actin cytoskeleton and the cell nuclei. Dilutions used for these stainings were: 1:800 for the monoclonal anti-vinculin antibody, 1:100 for the FITC-Phalloidin 488, 1:500 for Hoechst and 1:400 for the goat antimouse AlexaFluor 555- conjugated antibody. Finally the samples were washed with PBS, followed by mQ water and mounted with cover glass in Mowiol (Sigma-Aldrich, 10852-250G), 
then viewed and photographed in an inverted fluorescent microscope (Axio Observer ZI, Zeiss). At least three representative images were captured for each sample at low (10x) magnification for overall morphology and at high (63x) magnification to view focal adhesions and cytoskeleton organization.

\section{Fibronectin matrix secretion}

ADMSCs, as harvested from each passage, were seeded at a concentration of $1 \times 10^{4}$ cells $/ \mathrm{cm}^{2}$ onto the pre-coated with FN standard $20 \times 20 \mathrm{~mm}$ glass coverslips placed in a sterile $30 \mathrm{~mm}$ Petri dish (Sigma-Aldrich, P5471-500EA). The samples from the different passages (as indicated in the text) were further cultured for 3 days in basal medium with $10 \% \mathrm{FBS}$, then fixed, permeabilized and stained with polyclonal anti fibronectin (FN) antibody (Abcam, ab2413) followed by goat anti-rabbit IgG AlexaFluor 488 (Life Technologies, California, USA, A-11008) as secondary antibody. Dilutions used were: 1:500 for polyclonal anti FN antibody and 1:400 for Goat anti- Rabbit IgG, both incubated for 30 min at $37^{\circ} \mathrm{C}$. Finally, samples were mounted and viewed for fluorescence, as above.

FN matrix formation was quantified using FUJI (ImageJ) software as follows. Micrographs were adjusted, and the main green value was measured for five different "background" areas (i.e., not covered by the FN matrix). Then, a threshold was set matching the measured mean grey values and all the pixels above the threshold were counted. Further, counted pixels were plotted as a percent of the total pixels per each microscopic field.

\section{Senescence}

To identify the senescent cells, a Senescence Cells Histochemical Staining Kit (Sigma, CS0030) was used providing conditions for rapid staining for beta-galactosidase. 1 x $10^{4}$ cells were seeded on FN coated PNIPAM and control TC plates and cultured for 3 days in basal 
medium with $10 \%$ FBS. Following the manufacturer protocol, the samples were fixed for 7 minutes then incubated with the Staining Mixture at $37{ }^{\circ} \mathrm{C}$ overnight. $\mathrm{CO}_{2}$ was not used as the staining of senescent cells is $\mathrm{pH}$ dependent. Afterwards, the cells were washed with PBS. To count the total amount of beta-galactosidase positive senescent cells (blue stained). The samples where observed at a Nikon Eclipse e800 microscope combining with the phase contrast to view the non-stained cells. In some cases, characteristic images were acquired. To assure accuracy, the measurements were performed in triplicates and, when necessary, counted by two independent observers.

\section{Differentiation of ADMSCs}

For these experiments we used ADMSCs from early passage 4 (p4) to exclude the possible effect of ageing on the asymmetric cell division. The osteogenic differentiation was monitored by following the ALP activity and mineralization, as detailed below.

ALP activity was determined at days 3, 7 and 21 in triplicated samples using 24 well plates. The cells were lysed with $200 \mu \mathrm{L}$ of M-PER (Mammalian Protein Extraction Reagent, Pierce,78501) per sample and after 5 min of shaking the lysates were centrifuged in Eppendorf centrifuge (14000g for $5 \mathrm{~min})$. The supernatants were transferred in a 96 well plate followed by addition of alkaline buffer (Sigma-Aldrich, A9226), distilled water $\left(\mathrm{dH}_{2} \mathrm{O}\right)$ and Phosphate substrate (Sigma-Aldrich, P5994) according the manufacturers protocol. The plates were incubated for $5 \mathrm{~min}$ at $37{ }^{\circ} \mathrm{C}$ in dark and $3 \mathrm{M} \mathrm{NaOH}$ was used to stop the reaction before measuring the adsorption at $405 \mathrm{~nm}$ using Microtiter plate reader. Standards prepared form $\mathrm{p}$ nitrophenol (10 mM, Sigma-Aldrich, 7660) solutions in the concentration range $6 \times 10^{-2} \mathrm{mM}$ to $1.0 \mathrm{mM}$ were used to prepare a calibration curve.

Mineralization was studied using Alizarin red staining/extraction protocol. Briefly, at day 3, 7 and 21 triplicated samples were fixed with $4 \%$ paraformaldehyde (10 min) and after 
washing with $\mathrm{dH}_{2} \mathrm{O}, 1 \mathrm{~mL}$ Alizarin red $\mathrm{S}$ solution (40 mM, pH 4.2, Sigma-Aldrich, A5533) was added to each well. The staining solution was removed after $10 \mathrm{~min}$ and the well were washed 5 times with $\mathrm{dH}_{2} \mathrm{O}$ and air-dried at room temperature. The amount of matrix mineralization was quantified by dissolving the bound Alizarin red S to each sample in $10 \%$ Cetylpyridinium chloride (Serva, 426900) for $15 \mathrm{~min}$ at room temperature before measuring the absorption at $470 \mathrm{~nm}$ with plate rider as above. Standards prepared form Alizarin $\mathrm{S}$ red solutions in the concentration range $3.9 \times 10^{-} 2 \mathrm{mM}$ to $2.5 \mathrm{mM}$ were used to produce a calibration curve.

\section{Statistical analysis}

Statistical significance was determined by one-way ANOVA test using Sigma Plot Scientific Data Analysis and Graphing Software. Significant differences were determined using Post hoc analysis (Bonferroni t-test). Values with $\mathrm{p}<0.05$ were considered as significantly different. All quantitative data in the figures are presented as mean \pm standard deviation (SD).

\section{RESULTS AND DISCUSSION}

In this study we created a model DAE adding FN to the system as coating on the top of smart thermo-responsible PNIPAM surfaces. After applying periodic temperature changes (twice a day) on the adhering ADMSCs, we basically followed their morphology and symmetric and asymmetric division with added focus on their ageing. The rationale behind was to look for a system that might provide control over the stem cells fate through application of external physical stimuli. Basically, we introduced FN in this study motivated from the recently established role of this protein for the maintaining of stem cells in the niche $[14,23]$. 
More specifically, three were the main issues targeted in this work. First, (i) monitoring the morphological changes of ADMSCs upon single temperature cycle, including focal adhesions formation, actin cytoskeleton development and overall cell shape. Secondly, (ii) the study of the functional response of ADSCs during symmetric division at FN-DAE. For this purpose, we studied proliferation (i.e., the cell doubling time), senescence, cell size and FN matrix secretion as function of increasing passage number - all of them indirect signs for stem cells ageing. Finally, (iii) we studied the osteogenic response of ADMSCs using early p4 passage (to avoid consequences of ageing) to assess the eventual changes in their asymmetric division targeting the particular effect of FN-DAE.

The overall design of the experiments is presented in Figure 1.

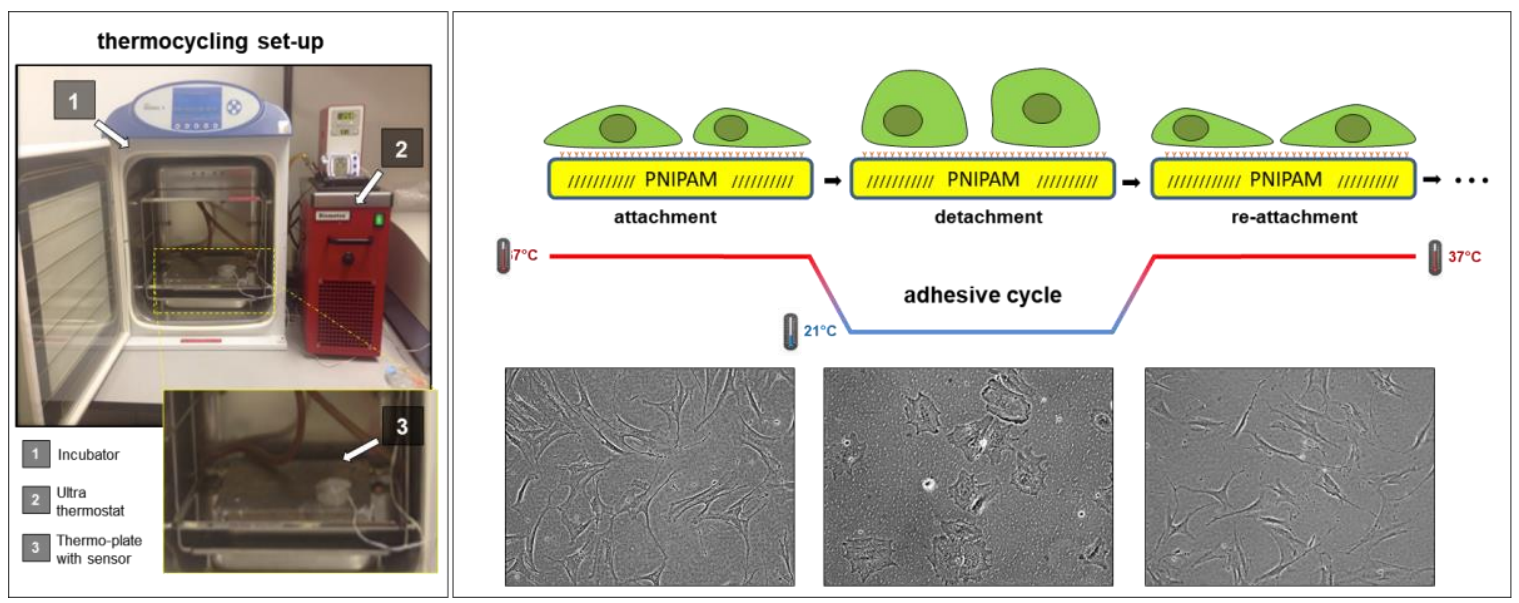

Figure 1 Overall design of the experiments. Upon varying the temperature, ADMSCs receive adhesive perturbations when cultured on FN-coated PNIPAM substrates.

Using a specially designed thermoplate fitted with circulating water jacket (Fig. 1 left) we periodically altered the culture temperature (manually, twice a day) by applying a temperature cycle below and above LCST (i.e., $37{ }^{\circ} \mathrm{C}-20^{\circ} \mathrm{C}-37^{\circ} \mathrm{C}$ ). Basically we followed a previously established protocol [26] showing that cooling time of $30 \mathrm{~min}$ at $20^{\circ} \mathrm{C}$ is sufficient 
to induce cell rounding (Figure 1 right), but without complete detachment of cells from the substrate. The adhesive cycle completes upon increasing the temperature to $37^{\circ} \mathrm{C}$ when the cells reattach and spread over the substrate - a process that takes further 30 min. Thus, during the temperature cycle of $1 \mathrm{~h}$ the ADMSCs receive an adhesive (mechanical) perturbation, but do not detach completely from the substratum.

\section{ADMSCs show reversible spreading during single temperature cycle}

To explore the DAE toward FN, we first needed to confirm the reversibility of the attachment-detachment process when ADMSCs were cultured on FN-coated PNIPAM substrate. It is noteworthy that the attachment of ADMSCs was performed in serum-free medium, thus assuring single-protein attachment conditions (see Methods Section). As displayed in Figure 2, the adhered cells showed elongated actin filaments (green) and well developed vinculin containing focal adhesions (red) as seen in the augmented insets. This morphology was characteristic for both FN-coated PNIPAM (Figure 2D) and control polystyrene (Figure 2A) samples when cultured at $37^{\circ} \mathrm{C}$. However, while the morphology of ADMSCs in controls remained unaltered upon lowering the temperature to $20{ }^{\circ} \mathrm{C}$ (Figure $2 \mathrm{~B}$ ), it caused significant rounding of cells on PNIPAM/FN samples, indicative for an initial cell detachment (Fig 2E). 

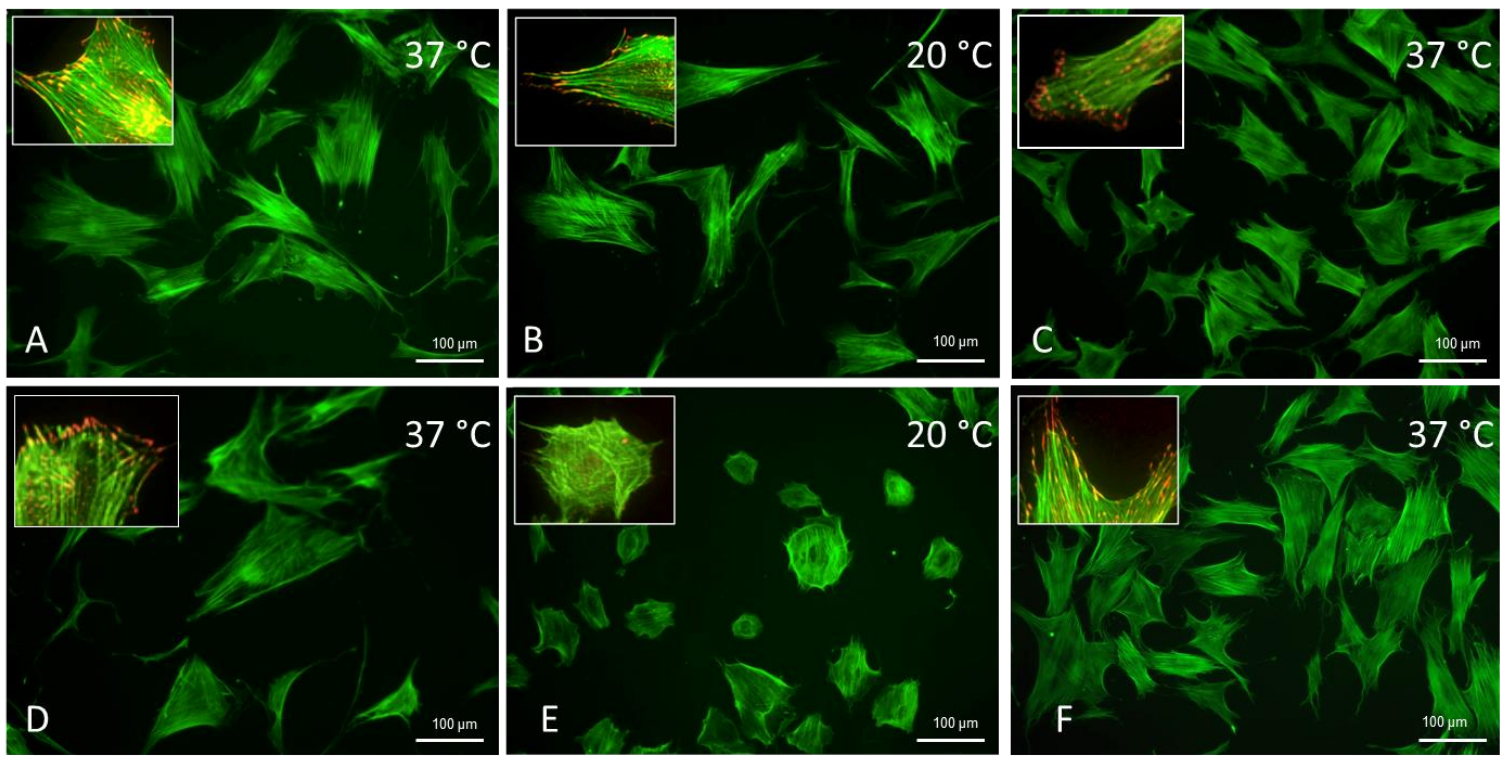

Figure 2 Morphological changes of ADMSCs during a standard thermo-cycle. A, B and C show the typical morphology of cells cultured on the control TC polystyrene, while D, E and F on FN-coated PNIPAM substrate. The cells were stained for actin and vinculin to view the development of actin cytoskeleton and focal adhesions. A and D show the cells after $3 \mathrm{~h}$ of incubation at $37{ }^{\circ} \mathrm{C}$; B and $\mathrm{E}$ after lowering the temperature to 20 ${ }^{\circ} \mathrm{C}$ for $30 \mathrm{~min}$ and $\mathrm{C}$ and $\mathrm{F}$ when the samples were reversed to $37{ }^{\circ} \mathrm{C}$ for $12 \mathrm{~h}$. Insets represent $3 \mathrm{X}$ augmented images of a typical cell for the given sample.

The partial detachment of cells was further confirmed by the disappearance of focal adhesions (inset of Figure 2E), which was not altered on control TC polystyrene (Figure 2B). As shown in Figure 2C, elevating the temperature back to $37^{\circ} \mathrm{C}$ resulted in cells re-attachment, consistent with the development of flattened cell morphology and the re-appearance of focal adhesion complexes.

The detachment of cells from thermo-responsive surfaces like PNIPAM is a welldescribed phenomenon, referring also to ADMSCs, as shown in our previous study [26]. The lowering of temperature below LSCT induces an increase of material surface hydration $[34,38,39]$, reduction of the attractive Van der Waals forces and a relative increase of the 
repulsive osmotic interactions [34]. All this lead to the release of adsorbed proteins (repelling effect), including the ECM constituents, and the cells detach together with them [38]. In our system it relates to the role of FN. As stated above, with our experimental setting (30 min cooling time) we induced only an initial detachment of cells, i.e., a partial release of FN, followed by a fast protein re-adsorption and re-attachment of cells upon rising the temperature to $37{ }^{\circ} \mathrm{C}$. We should consider, however, that the $\mathrm{FN}$ is already attached to the cells and presumably undergo substantial remodeling [24], which makes the interpretations more complex. Nevertheless, such re-attachment of whole cells layers upon transfer to another substrate was already demonstrated when thermo-responsive material were used for cell sheet engineering $[32,39]$. These studies showed that the re-attachment of whole cell sheets is due to the re-adsorption of the cells-secreted ECM proteins. We claim that this phenomenon works also for single cells and in a single-adhesive protein (i.e., FN) system, as explained in this work. Hence, the re-adsorption of FN together with the cells on the top of the PNIPAM substrate is the working mechanism behind our engineered DAE toward FN.

\section{Cell growth and FN secretion}

The impact of FN on ADMSCs self-renewal (symmetric growth) at DAE was further examined. FN-coated TC polystyrene was used as positive control and processed in the same way as PNIPAM samples to exclude the eventual metabolic effects of temperature. The cells were cultured for 5 days (two thermocycles per day), then harvested and counted before seeding them again at the same density for the next passage, and the process continued up to $\mathrm{p} 12$. The cells yield at each passage was used to calculate the CPDN and to perform all the morphological studies. As shown in Figure 3, a substantial effect of FN/DAE on the growth kinetic of ADMSCs was observed. More specifically, we found that FN significantly promotes the symmetric self-renewal of stem cells, particularly between $\mathrm{p} 5$ and $\mathrm{p} 8$. Afterward, the growth kinetics sharply decline making cell numbers undistinguishable from the controls for p10 and 
$\mathrm{p} 11$, followed by a significant inhibition at $\mathrm{p} 12$ for all samples, presumably due to ageing processes. This result differs from those obtained in our previous study [26], where no significant benefits of thermo-cycling was observed using "classical" (i.e., FN-free) TC conditions. As stated above, FN is omitted from the commercial serum preparations [35], therefore we assume that the actual adhesive protein in this system was vitronectin $[35,36]$. Consequently, it might be presumed that the periodic alteration of vitronectin bonds are not important for the symmetric renewal of stem cells, apart from the clear stimulatory effect FN binding established in this study.

Collectively this result indicates that the periodic alterations of ADMSCs attachment to FN affects positively their self-renewal and therefore may be implicated for producing higher amounts of stem cells for clinical applications. 


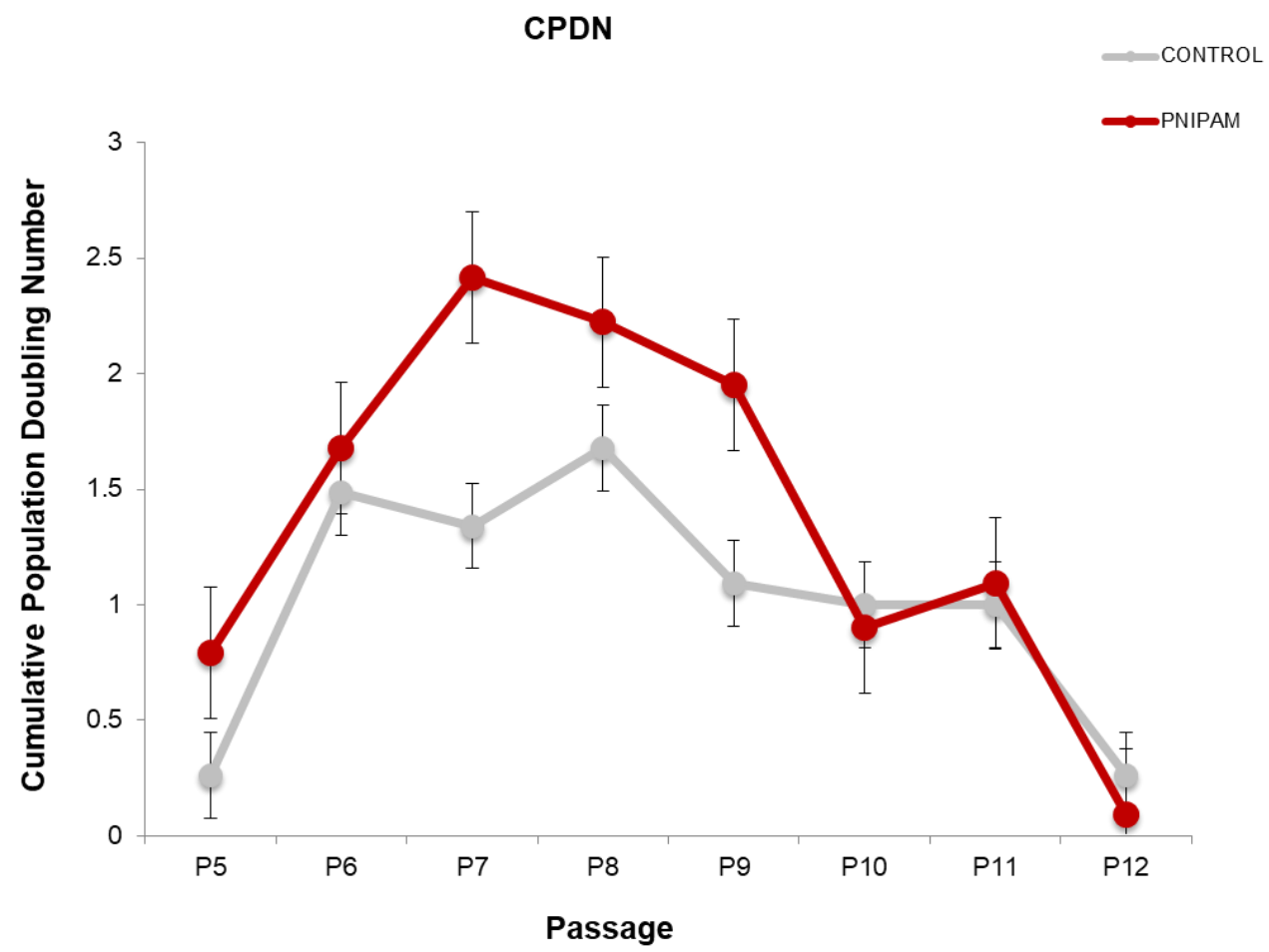

Figure 3 Proliferation rate of ADMSCs cultured under periodic thermocycling on FN-coated PNIPAM and control TC polystyrene with progressive passage numbers (from $\mathrm{p} 5$ to $\mathrm{p} 12$ ). The graph represents the CPDN calculated for each cell population (mean $\pm \mathrm{SD})$.

\section{Signs for Ageing of ADMSCs}

\section{Cell size}

For some of the passages we measured the average size of ADMSCs (Figure 4) in suspension considering that ageing of cells alters may their dimensions [40,41]. 


\section{Average Cell Size per Passage}

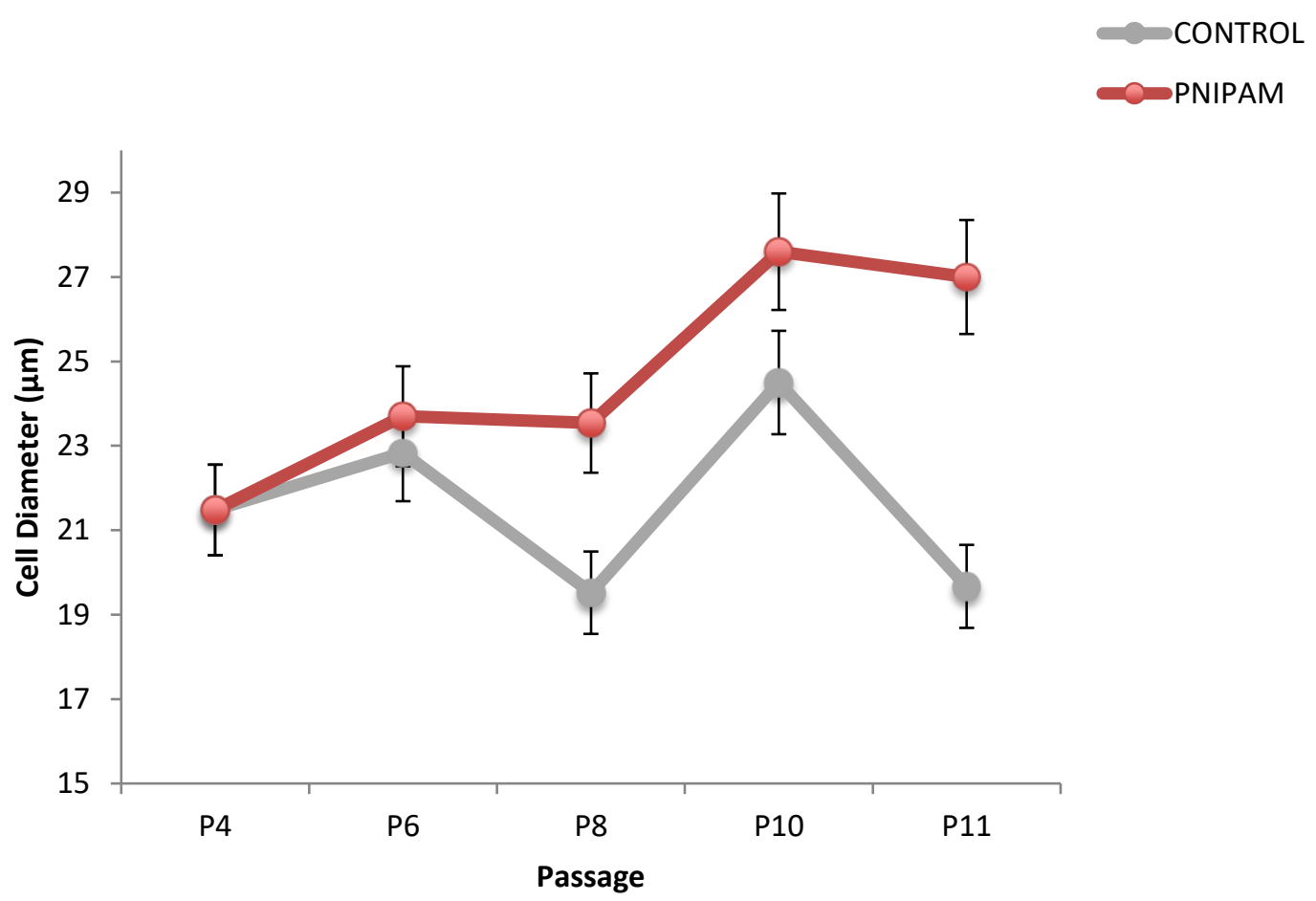

Figure 4 Average cell size obtained for different passages of ADMSCs cultured on FN-coated PNIPAM (red) and control TC Polystyrene (grey) under a dynamic adhesive environment.

We did not observe significant changes in the main diameter of ADMSCs at early p4-p6 passages, but afterwards it slowly increased at FN/DAE with progressing the passages number, raising from $21 \pm 2 \mu \mathrm{m}$ at $\mathrm{p} 4$ up to $28 \pm 3 \mu \mathrm{m}$ at $\mathrm{p} 11$. The mean cell size in controls, though varying from passage to passage, was permanently lower. The reason for this significant increase in the cell size at FN/DAE is not clear. It could be related to the accumulation of larger cells in interphase (G1-G2 stage) [42]. Such accumulation of pre-mitotic cells however cannot explain all our results, as generally no correlation with the cell growth kinetics was observed. For example, at p8 where ADMSCs proliferation was significantly higher with respect to the control (Figure 3), the cells were significantly larger. Conversely, at p11 where no difference 
in the proliferation was observed, the cells were still significantly larger on the DEAE/FN substrate (Figure 4).

Detailed morphological analysis performed in vitro show that stem cells generally diminish their size (and spreading area) with advancing the passages number [43], which correlates with shortening of the telomerases length, reduced protein synthesis and decline in their growth and differentiation potential - all characteristics of an in vitro ageing $[7,8,40]$. In fact, our data confirm this trend for the reduction in size, but only for control samples, showing approximately a $30 \%$ reduction in the main cell diameter, valid from p6 to p11 (Figure 4). The deviations at p8 (decrease in cells size) and p10 (increase over the initial size), may be explained with the influence of cell proliferation, which may affect the ratio between growing and resting cells. Indeed, at p8 (control samples) the proliferation was the highest, while at p10 it sharply declined (Figure 3), which correlates with the putative depletion or accumulation of larger pre-mitotic cell, respectively. The trend with DAE samples however was independent on the proliferation. Despite there exist some conflicting results concerning the size of ADMSCs at late passageing [44], the obtained tendency of cell enlargement could be explained with a constitutive accumulation of resting pre-mitotic cells, and consequently attributed to a kind of anti-ageing effect of FN at DAE. In this context, we can refer again to the studies of [14] where FN was characterized as a preferred adhesion substrate for the muscle stem cells involving $\beta 1$ integrin signalling pathway. A strong deregulation in this signalling mechanism was further found in aged mice, resulting in an insufficient attachment to $\mathrm{FN}$ in the niche. Interestingly, the reconstitution of FN level remobilizes stem cells in the niche and restores youth-like muscle regeneration [14]. Taken together with our data, these studies lead to the conclusion that DAE toward FN, as recapitulated ex vivo in our artificial niche, represents a novel anti-ageing mechanism. However, it has to be distinguished from the chronological ageing of the stem cells at the organismal level, which runs significantly slower [43]. 


\section{Senescence}

Another sign for ageing of stem cells is the appearance of positive for betagalactosidase senescent cells. Senescence is characterized by irreversible cell cycle arrest usually induced by shortening of telomeres [8]. Increasing evidences suggest that senescence is also a cause of stem cell ageing [10], which was the reason to perform such study also in our system.

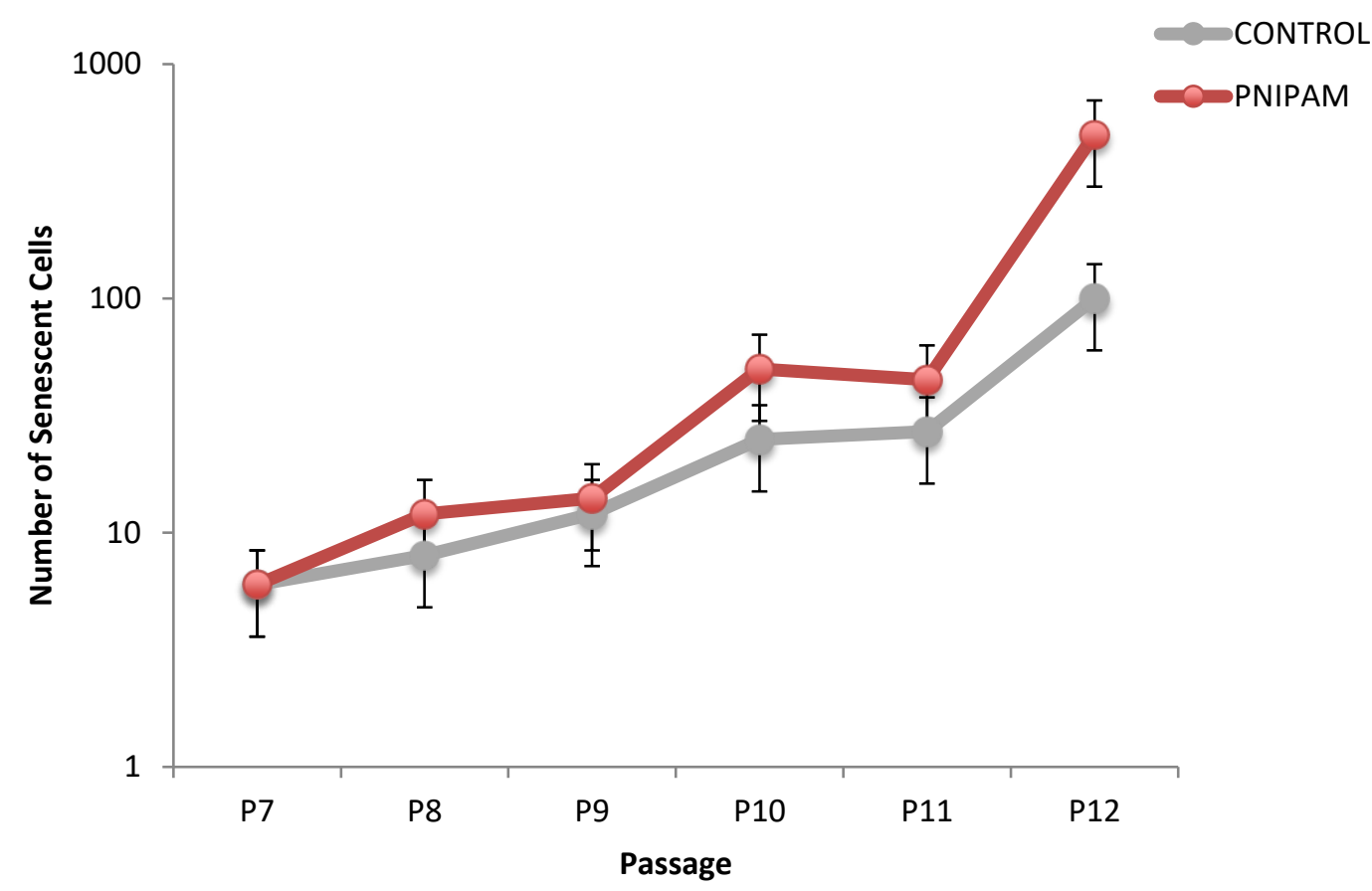

Figure 5 Main number of senescent cells at different passages. ADMSCs were cultured on FN-coated PNIPAM (red) and control TC polystyrene (grey). The total amount of beta-galactosidase positive cells (blue stained) was counted per each sample in triplicated experiment.

Figure 5 displays the total number of obtained senescent cells (per sample) as a function of passage number. The study involved passages $\mathrm{p} 7-\mathrm{p} 12$, where $\mathrm{p} 7-\mathrm{p} 9$ represent the samples with maximal cell growth, while in p10-p12 it was significantly reduced (as already discussed 
above and introduced in Figure 3). No differences related to the amount of senescence were found comparing FN-coated PNIPAM and control samples. The number of beta-galactosidase positive cells gradually increased from $\mathrm{p} 7$ to $\mathrm{p} 12$ on both samples consistent with the advancing of the ADMSCs ageing. It shows relative independency from the cell growth, and also from the adhesive perturbations. A slight (nonsignificant) tendency for higher number of betagalactosidase positive cells on the PNIPAM samples was observed, particularly in the last p12 passage, involving approximately the $12 \%$ from the total population (with respect to the $8 \%$ in p12 control), suggesting that FN/DAE might promote some accumulation of senescent cells. It has to be considered however that the origin of beta-galactosidase expression and its cellular roles in senescence are not known [45], therefore some other mechanisms that lie behind this process cannot be excluded.

\section{Fibronectin matrix secretion}

The secretion of FN matrix is another indicator for ADMSCs functionality [1], known to be negatively affected by ageing [40]. Indeed, as can be observed in Figure 6, the FN secretion generally reduces with the progress of passages on both DAE and control samples, when compared for .example the early p4 (Figure 6 A and C) and the late p12 (Figure 6 B and D) passages. 

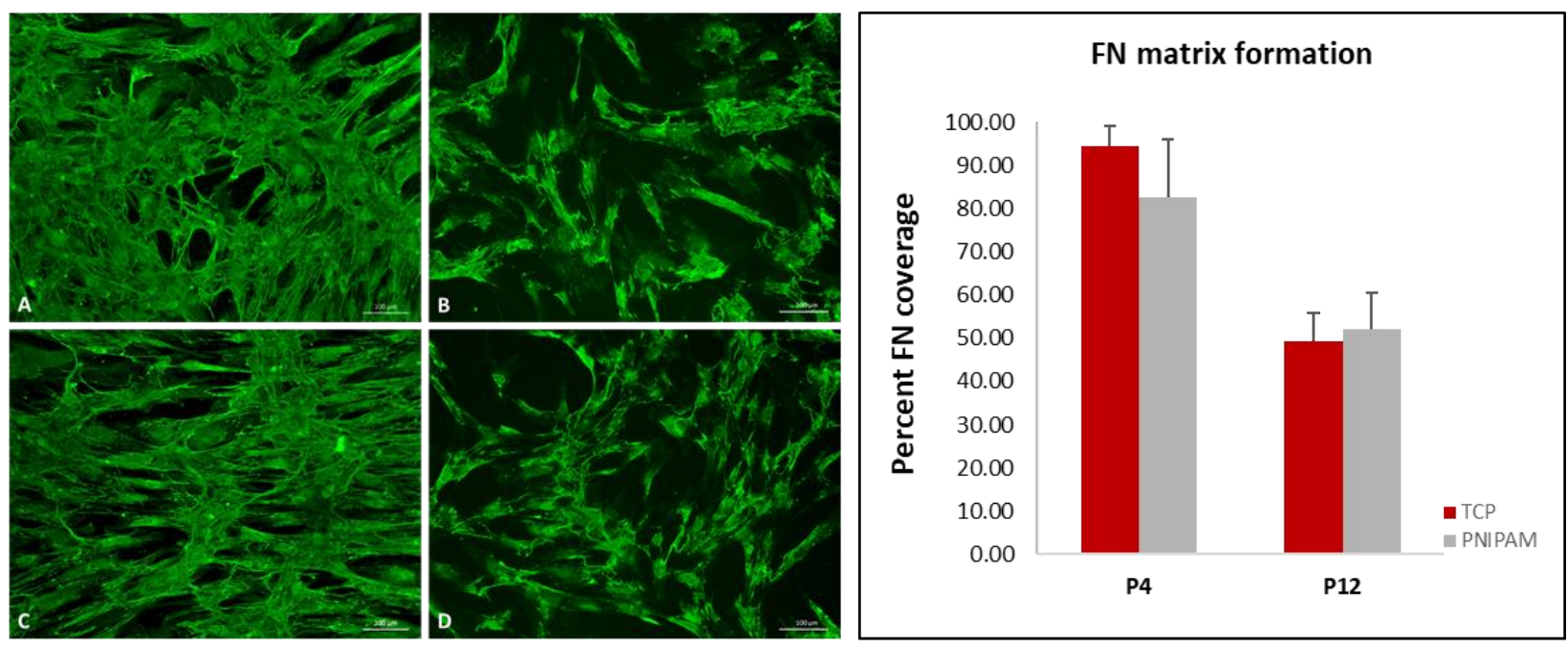

Figure 6 Fibronectin matrix secretion (green) of ADMSCs from early p4 (A, C) and late p12 (B, D) passages cultured on FN-coated PNIPAM (C-D) or control TC Polystyrene (TCP) (A-B) surfaces under periodic temperature perturbations. Right panel represents our attempt to quantify the FN matrix deposition using FUJI (ImageJ) software. Results are presented in "\% FN Coverage" of substratum where counted pixels above the threshold for the substratum alone were plotted as a percent of the total pixels per each microscopic field.

Though not very well pronounced difference, it is worthy to mention that FN secretion at late p12 was a bit higher on PNIPAM substrates, compared to the corresponding control polystyrene (Figure 6 D vs. B, respectively). Given that the initial FN deposition at early p4 shows rather an opposite trend (i.e., higher FN formation on control TCP plates), it suggests that DAE itself may supports FN matrix formation. The quantitative measurement of FN matrix deposition using FUJI (Image J) software generally confirmed this tendency for higher matrix formation on PNIPAM substrata at late p12 passage, however we could not observe statistically significant differences $(\mathrm{p}>0.05)$. 


\section{Differentiation of ADMSCs}

When cultured in osteogenic medium, ADMSCs follow three well-distinguished maturational phases consisting of proliferation, matrix maturation and mineralization [46]. Alkaline phosphatase activity (ALP) is a prerequisite for the mineralization of bone and thus represents an early biochemical marker for the osteogenic response of ADMSCs [15]. Therefore it peaks earlier, within 2 weeks before the mineralization take place [46].

To better characterize the osteogenic differentiation of ADMSCs with FN/DAE, we quantified both the ALP activity and the Ca deposition at different time intervals of culture. Cellular ALP activity was quantified at day 1, 7 and 14, with an expectations to have the typicaly higher expression within 2 weeks. Indeed, as shown in Figure 7, the ALP gradually increased in all samples, with maximal activity at day $14(\mathrm{p}<0.05)$, while negligible values were found at day 1 .

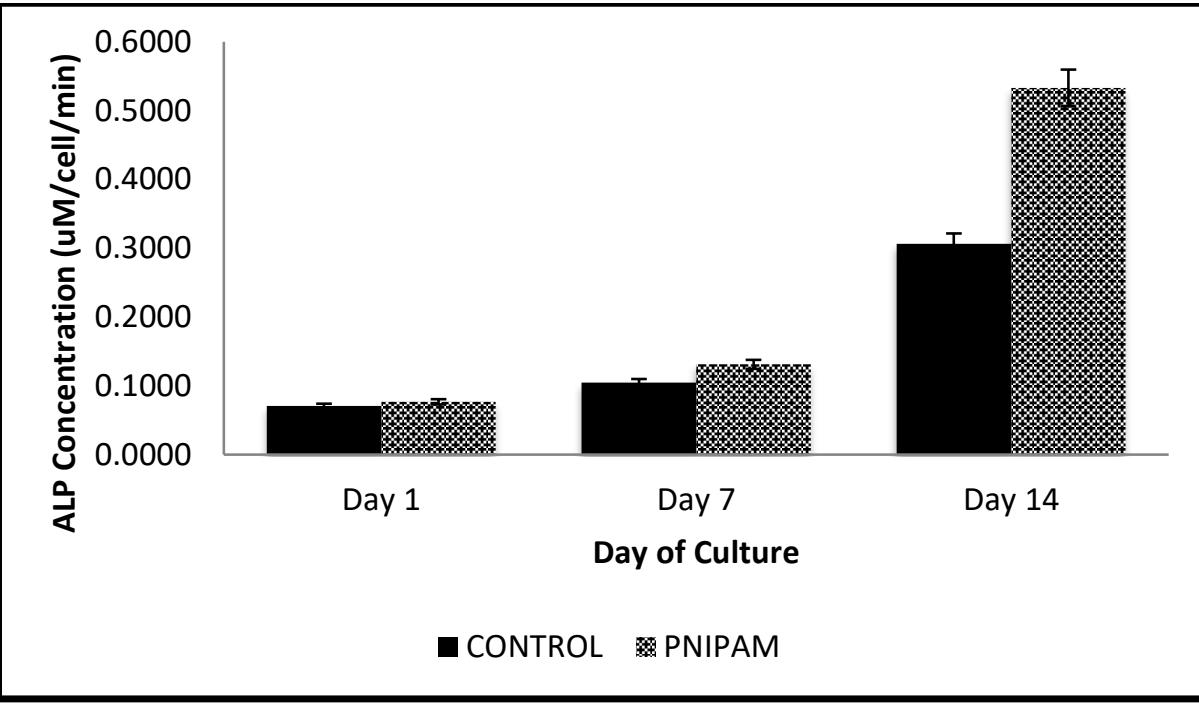

Figure 7 ALP activity of ADMSCs from early passage 4 at day 1, 7 and 14 of culture on control FNcoated polystyrene (blue) and FN-coated PNIPAM samples (red). 
ALP activity start to increase at day 7 , but with lower activity in control samples - a trend continuing up to day 14, when it significantly increased on FN-coated PNIPAM (about twice as much) compared to control surface $(\mathrm{p}<0.05)$.

One can assume that the lowered ALP activity in controls might be due to the metabolic stress of ADMSCs from the periodic temperature changes. These wrong conclusions may derive also from the fact that PNIPAM itself promotes (slightly) the osteogenic response of stem cells [47]. It was not the case however in our previous investigation [26] where, on the contrary, a strong inhibition of the osteogenic response of ADMSCs on plain PNIPAM substrata was observed upon periodic DEA perturbation.

The effect of FN at DAE is a new observation that must be attributed to the role of FN bond instability upon cyclic temperature perturbations. It has to be noted that this result differ significantly from our previous study where "classical" (i.e., FN-free) culturing conditions were used [26]. This means that the adhesion of ADMSCs to the PNIPAM substrate was dependent on other attachment factors that contained in the serum $[35,36]$. However, here we utilized a single protein system where the adhesive factor is definitely FN, suggesting that the anchorage of ADMSCs to FN is essential for the maintaining of their differentiation phenotype. It correlates also with the earlier observation of [48] showing direct link between the $\beta 1$ integrin expression (i.e., the main FN receptor) and the recruitment of osteoblast-like cells involved in the interleukin-1 induced ALP activity.

Such stimulatory effect of FN however was not observed in respect to the Ca deposition, characteristic for a later stage of mineralization. 


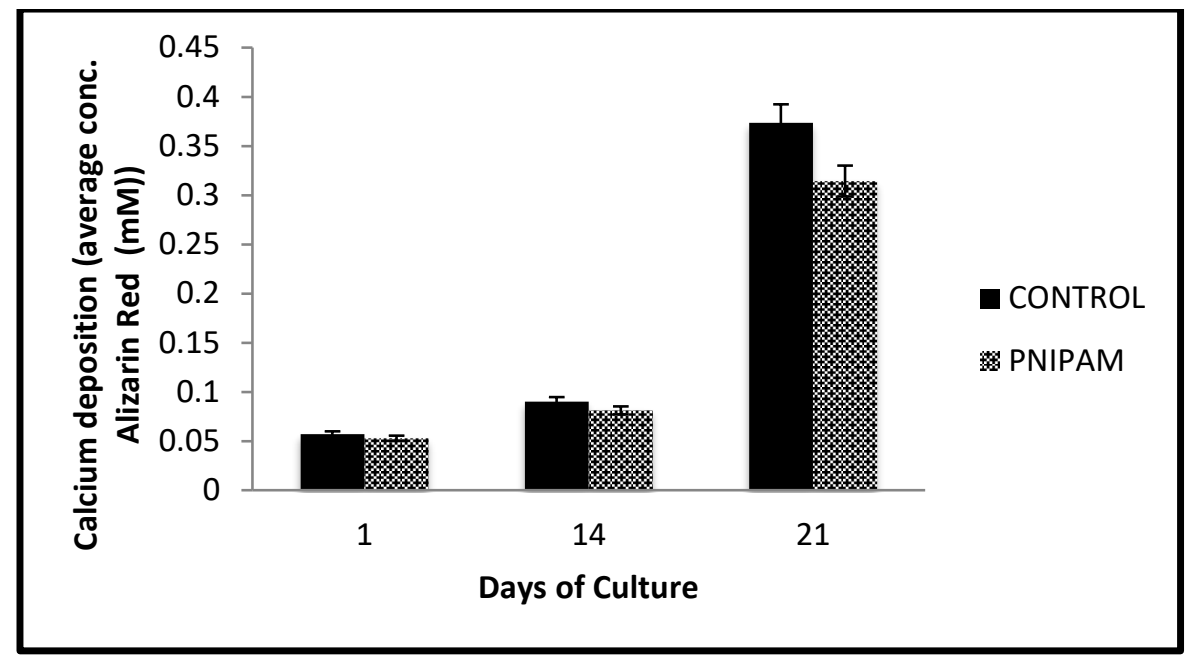

Figure 8 Calcium deposition of ADSCs on control FN-coated polystyrene and FN-coated PNIPAM samples as measured by Alizarin red staining/extraction at days 1, 14 and 21 .

As shown in Figure 8, though the mineralization sharply increase at day 21 (at day 14 only slight elevation was detected), a minimal difference in Alizarin red uptake in PNIPAM-FN samples versus the control was observed.We assume that the Ca deposition (showing here even an opposite trend) is a process independent on the stem cells attachment to FN, therefore the adhesive perturbations are ineffective. It should be noted however that $\mathrm{Ca}$ deposition on PNIPAM still runs, as do on control polystyrene plates, but it is just not affected by DAE. We accept that calcification is a relatively "static" process, which is not necessarily dependent on DAE toward FN. Conversely, the process of early bone formation mediated by BMP signalling events [49], that involves the initial ALP activation and is required for the differentiation of osteoblasts from stem cell precursors [46], clearly corroborate with our results showing significant increase of ALP activity at DAE-FN (Figure 7). We argue, however, that the subsequent mineralization process is dependent rather on Ca signalling events than on the cellECM interaction.

Although many studies on the osteogenic stem cells differentiation have been performed, the molecular mechanism of Ca-mediated signalling is still not sufficiently elucidated [46,50-52]. 
Nevertheless, the initial phase of mineralization includes the formation of $\mathrm{Ca}^{2+}$ ions and inorganic phosphate $(\mathrm{Pi})$ within mineral vesicles [50]. The second phase require interaction of these vesicles with the ECM, involving mostly type I collagen [19,51]. The breakdown of vesicles, expose the preformed HA to the extracellular fluid [50], which triggers the osteogenic genes activation (SMAD and RAS family) assuring the later stages of differentiation [53]. All these events however have nothing to do with the adhesion of stem cells to FN.

Nevertheless FN is important for the function of osteogenic precursors. Bone formation requires recruitment of ASCs at the place of injury. The role of FN in this process is confirmed by the fact that integrin receptor $\alpha 5 \beta 1$ (i.e., the main $\mathrm{FN}$ receptor) is compulsory for the initial bone-like nodule formation [54]. The homing of stem cells in the niche also depends on their proper anchorage, which again highlights the role of the adhesive interaction with the ECM molecules [55,56], one of which obviously is FN [14,55,57]. In agreement with this are also the already mentioned studies showing that the specific adhesion to FN recruit stem cells in the niche $[9,14,23]$, moreover in an age-related manner [14]. Therefore it might argued that stem cell niche itself represents a dynamic adhesive environment on which ADMSCs are obviously well adapted and respond on it

\section{CONCLUSIONS}

In an attempt to highlight the role of physical cues on the stem cells behaviour, here we established a significant effect of FN on the ADMSCs behaviour at DAE, through culturing them on PNIPAM substrates undergoing periodic temperature changes. We found that the addition of FN to the system substantially increased the symmetric renewal and the initial osteogenic response of stem cells. The effect of this engineered DEA on symmetric division of ADMSCs was evident by the significantly increased cells yield between $\mathrm{p} 6$ and p9. Afterwards, 
it dropped down for all the samples, consistent with the increased number of senescent cells, the average cell size and the reduced secretion of FN matrix - all characteristics of stem cell ageing. DAE involving FN tended to improve most of the above ageing parameters. Osteogenic response of ADMSCs was also enhanced judged by the significant increased ALP activity at day 7 and 14. Ca deposition, however, was not affected suggesting only a partial effect of FN binding involving the early stages of ADSCs differentiation.

These findings, in addition to the renewed ability to engineer an ex-vivo stem cell niche-

like environment, could create multiple opportunities for obtaining higher amounts of functional stem cells for clinical application. Although higher ALP expression at DAE-FN is not sufficient alone to indicate significant osteogenesis, it serves as a positive marker in this direction. Future research work should be addressed to the use of more advanced techniques (e.g., qPCR) for the quantification of ADMSCs differentiation at higher passages.

\section{ACKNOWLEDGMENTS}

This work was mainly supported by the European Commission through FP7 IndustryAcademia Partnerships and Pathways (IAPP) project FIBROGELNET. The valuable contribution of project MAT 2015-69315-C3 MYOHEAL with the Spanish Ministry of Science and Innovation and CIBER-BBN are also acknowledged. FA acknowledges the partial support of FWF under Lise Meitner program (M-1777) and grant No 713690 provided by European Commission within the Horizon 2020 Marie Curie-Skłodowska program.

\section{REFERENCES}


[1] Krishnamoorthy, N. et al. (2017). A novel strategy to enhance secretion of ECM components by stem cells: Relevance to tissue engineering. Tissue Engineering Part A, 24, 145156.

[2] Ferraro, F. et al. (2010). Adult stem cells and their niches. Advances in Experimental Medicine and Biology, 695, 155-168.

[3] Frese, L. et al. (2016). Adipose tissue derived stem cells in regenerative medicine. Transfusion Medicine and Hemotherapy, 43, 268-274.

[4] Caplan, A.I. (1991). Mesenchymal stem cells. Journal of Orthopaedic Research, 9, 641650.

[5] Caplan, A.I. (2005). Mesenchymal stem cells: cell-based reconstructive therapy in orthopedics. Tissue Engineering, 11, 1198-1211.

[6] Prockop, D.J. (1997). Marrow stromal cells as stem cells for nonhematopoietic tissues. Science, 276, 71-74.

[7] Baker, N. et al. (2015). Characterization of bone marrow-derived mesenchymal stem cells in ageing. Bone, 70, 37-47.

[8] Boyette, L.B. et al. (2014). Adult stem cells and diseases of ageing. Journal of Clinical Medicine, 3, 88-134.

[9] Sousa-Victor, P. et al. (2015). Muscle stem cell ageing: regulation and rejuvenation. Trends in Endocrinolology \& Metabolism, 26, 287-296.

[10] Kennedy, B.K. et al. (2014). Geroscience: linking ageing to chronic disease. Cell, 159, 709-713.

[11] Reitinger, S. et al. (2015). Systemic impact molds mesenchymal stromal/stem cell ageing. Transfusion and Apheresis Science, 52, 285-289. 
[12] Scadden, D.T. (2006). The stem-cell niche as an entity of action. Nature, 441, 10751079.

[13] Phadke, A. et al. (2010). Functional biomaterials for controlling stem cell differentiation. In: Biomaterials as Stem Cells Niche, Krishnendu R. Ed., Springer-Verlag, Berlin Heidelberg.

[14] Lukjanenko, L. et al. (2016). Loss of fibronectin from the aged stem cell niche affects the regenerative capacity of skeletal muscle in mice. Nature Medicine, 22, 897-905.

[15] Birmingham, E. et al. (2012). Osteogenic differentiation of mesenchymal stem cells is regulated by osteocyte and osteoblast cells in a simplified bone niche. European Cell Mater, 23, $13-27$.

[16] Kuang, S. et al. (2008). Niche regulation of muscle satellite cell self-renewal and differentiation. Cell Stem Cell, 2, 22-31.

[17] Morrison, S.J. et al. (2006). Asymmetric and symmetric stem-cell divisions in development and cancer. Nature, 441, 1068-1074.

[18] Teixeira, A. et al. (2010). Designing and engineering stem cell niches. MRS Bulletin, $35,591-596$.

[19] Donzelli, E. et al. (2007). Mesenchymal stem cells cultured on a collagen scaffold: In vitro osteogenic differentiation. Archives of Oral Biology, 52, 64-73.

[20] Salasznyk, R.M. et al. (2004). Adhesion to vitronectin and collagen I promotes osteogenic differentiation of human mesenchymal stem cells. Journal of Biomedicine and Biotechnology, 1, 24-34.

[21] Schneider, R.K. et al. (2010). Osteogenic differentiation of adult bone marrow and perinatal umbilical mesenchymal stem cells and matrix remodelling in three-dimensional collagen scaffolds. Biomaterials, 31, 467-480. 
[22] Somaiah, C. et al. (2015). Collagen promotes higher adhesion, survival and proliferation of mesenchymal stem cells. PLoS One, 10, e0145068.

[23] Singh, P. et al. (2012). Fibronectin and stem cell differentiation - lessons from chondrogenesis. Journal of Cell Science, 125, 3703-3712.

[24] Trappmann, B. et al. (2012). Extracellular-matrix tethering regulates stem-cell fate. Nature Materials, 11, 642-649.

[25] Gattazzo, F. et al. (2014). Extracellular matrix: A dynamic microenvironment for stem cell niche, Biochimica and Biophysica Acta, 1840, 2506-2519.

[26] Bianchi, M.V. et al. (2017). Dynamic adhesive environment alters the differentiation potential of young and ageing mesenchymal stem cells. Materials Science and Engineering C, $78,467-474$.

[27] Zhang, W. et al. (2013). Comparison of the use of adipose tissue-derived and bone marrow-derived stem cells for rapid bone regeneration. Journal of Dental Research, 92, 11361141.

[28] McLaughlin, M.M. et al. (2013). The use of adipose-derived stem cells as sheets for wound healing. Organogenesis, 9, 79-81.

[29] Cohen, S. (2010) Emerging applications of stimuli-responsive polymer materials. Nature Materials, 9, 101-113.

[30] Kikuchi, A. et al. (2004). Temperature responsive, polymer-modified surfaces for green chromatography. Macromolecular Symposia, 207, 217-227.

[31] Yamato, M. et al. (2000). Release of adsorbed fibronectin from temperature-responsive culture surfaces requires cellular activity. Biomaterials, 21, 981-986.

[32] Da Silva, R.M.P. et al. (2007). Smart thermoresponsive coatings and surfaces for tissue engineering: switching cell-material boundaries. Trends in Biotechnology, 25, 577-583. 
[33] Ward, M.A. et al. (2011). Thermoresponsive polymers for biomedical applications. Polymers, 3, 1215-1242.

[34] Okano, T. et al. (1995). Mechanism of cell detachment from temperature-modulated, hydrophilic-hydrophobic polymer surfaces. Biomaterials, 16, 297-303.

[35] Groth, T. et al. (1998). Altered vitronectin receptor (alpha-v integrin) function in fibroblasts adhering on hydrophobic glass. Journal of Biomedical Materials Research, 44, 341351.

[36] Toromanov, G. et al. (2015). Dynamic behavior of vitronectin at the cell-material interface. ACS Biomaterials Science \& Engineering, 1, 927-934.

[37] Greenwood, S.K.et al. (2004). Population doubling: A simple and more accurate estimation of cell growth suppression in the in vitro assay for chromosomal aberrations that reduces irrelevant positive results. Environmental and Molecular Mutagenesis, 43, 36-44.

[38] Yamada, N. et al. (1990). Thermo-responsive polymeric surfaces; control of attachment and detachment of cultured cells. Macromolecular Rapid Communications, 11, 571-576.

[39] Shimizu, K. et al. (2010). Oxygen plasma-treated thermoresponsive polymer surfaces for cell sheet engineering. Biotechnology and Bioengineering, 106, 303-310.

[40] Muniandy, M.K.R. et al. (2013). Proliferation rate and cell size analyses of human peripheral blood suspension stem cells from three culturing terms populations. Malaysian Applied Biology, 42, 59-63.

[41] Raven, P.H. et al. (2011). How cells divide. In: Biology 10th, McGraw-Hill, New York. [42] Holstein, T.W. (1990). Cell cycle of stem cells in hydra. Developmental Biology, 142, 392-400. 
[43] Geissler, S. et al. (2012). Functional comparison of chronological and in vitro ageing: Differential role of the cytoskeleton and mitochondria in mesenchymal stromal cells. PLoS One, 7, e52700.

[44] Legzdina, D. et al. (2016). Characterization of senescence of culture-expanded human adipose-derived mesenchymal stem cells. International Journal of Stem Cells, 9, 124-136.

[45] Lee, B.Y. et al. (2006). Senescence-associated $\beta$-galactosidase is lysosomal $\beta$ galactosidase, Ageing Cells, 5, 187-195.

[46] Gordon J. et al. (2011). Epigenetic regulation of early osteogenesis and mineralized tissue formation by a HOXA10-PBX1-associated complex. Cells Tissues Organs, 194, 146-150. [47] Shi D. et al. (2009). Proliferation and multi-differentiation potentials of human mesenchymal stem cells on thermoresponsive PDMS surfaces grafted with PNIPAM. Biosciences Reports, 30, 149-158.

[48] Dedhar, S. (1989). Signal transduction via the b1 integrins is a required intermediate in interleukin-1 b induction of alkaline phosphatase activity in human osteosarcoma cells. Experimental Cell Research, 183, 207-214.

[49] Zhao M. et al. (2002). Bone morphogenetic protein receptor signaling is necessary for normal murine postnatal bone formation. Journal of Cellular Biology, 157, 1049-1060.

[50] An, J. et al. (2016). Mineralization in hard tissues. In: Biomineralization and Biomaterials Fundamentals and Application, Aparicio C. and Ginebra M.P. Eds.

[51] Fakhry, M. et al. (2013). Molecular mechanisms of mesenchymal stem cell differentiation towards osteoblasts. World Journal of Stem Cells, 5, 136-148.

[52] Viti, F. et al. (2016). Osteogenic differentiation of MSC through calcium signaling activation: Transcriptomics and functional analysis. PLoS One, 11, e0148173. 
[53] Murshed, M. et al. (2005). Unique coexpression in osteoblasts of broadly expressed genes accounts for the spatial restriction of ECM mineralization to bone. Genes Development, 19, 1093-1104.

[54] Keselowsky, B.G. et al. (2007). Integrin $\alpha 5$ controls osteoblastic proliferation and differentiation responses to titanium substrates presenting different roughness characteristics in a roughness independent manner. Journal of Biomedical Materials Research Part A, 80, 700710.

[55] Hristova-Panusheva, K. et al. (2017). Age-related changes in adhesive phenotype of bone marrow-derived mesenchymal stem cells on extracellular matrix proteins. Journal of New Results in Science, 6, 11-19

[56] Nedjari, S. et al. (2017). Three dimensional honeycomb patterned fibrinogen based nanofibers induce substantial osteogenic response of mesenchymal stem cells. Scientific Reports, 7, 15947.

[57] Cosgrove, B.D. et al. (2010). A home away from home, challenges and opportunities in engineering in vitro muscle satellite cell niches. Differentiation, 78, 185-194.

[58] Chen, S. et al. (2013). Adhesion in the stem cell niche, biological roles and regulation. Development 15, 255-265.

\section{FIGURE CAPTIONS}

Figure 1 Overall design of the experiments. Upon varying the temperature, ADMSCs receive adhesive perturbations when cultured on FN-coated PNIPAM substrates.

Figure 2 Morphological changes of ADMSCs during a standard thermo-cycle. A, B and C 
show the typical morphology of cells cultured on the control TC polystyrene, while D, E and F on FN-coated PNIPAM substrate. The cells were stained for actin and vinculin to view the development of actin cytoskeleton and focal adhesions. A and D show the cells after $3 \mathrm{~h}$ of incubation at $37^{\circ} \mathrm{C} ; \mathrm{B}$ and $\mathrm{E}$ after lowering the temperature to $20{ }^{\circ} \mathrm{C}$ for $30 \mathrm{~min}$ and $\mathrm{C}$ and $\mathrm{F}$ when the samples were reversed to $37^{\circ} \mathrm{C}$ for $12 \mathrm{~h}$. Insets represent $3 \mathrm{X}$ augmented images of a typical cell for the given sample.

Figure 3 Proliferation rate of ADMSCs cultured under periodic thermocycling on FN-coated PNIPAM and control TC polystyrene with progressive passage numbers (from $\mathrm{p} 5$ to $\mathrm{p} 12$ ). The graph represents the CPDN calculated for each cell population (mean $\pm \mathrm{SD}$ ).

Figure 4 Average cell size obtained for different passages of ADMSCs cultured on FN-coated PNIPAM (red) and control TC Polystyrene (grey) under a dynamic adhesive environment.

Figure 5 Main number of senescent cells at different passages. ADMSCs were cultured on FNcoated PNIPAM (red) and control TC polystyrene (grey). The total amount of betagalactosidase positive cells (blue stained) was counted per each sample in triplicated experiment.

Figure 6 Fibronectin matrix secretion (green) of ADMSCs from early p4 (A, C) and late p12 (B, D) passages cultured on FN-coated PNIPAM (C-D) or control TC Polystyrene (A-B) surfaces under periodic temperature perturbations.

Figure 7 ALP activity of ADMSCs from early passage 4 at day 1, 7 and 14 of culture on control FN-coated polystyrene (blue) and FN-coated PNIPAM samples (red).

Figure 8 Calcium deposition of ADSCs on control FN-coated polystyrene and FN-coated PNIPAM samples as measured by Alizarin red staining/extraction at days 1, 14 and 21. 